\title{
Analysis of ultrasound field propagating through an inhomogeneous velocity layer
}

Tai-bao Li and Yasuhito Takeuchi

GE Yokogawa Medical Systems, Ltd.,

7-127, Asahigaoka 4-chome, Hino, 191 Japan

(Received 27 October 1993)

Keywords: Inhomogeneous velocity layer, Diffraction, Phase variation, Amplitude variation

PACS number: 43. 20. G

Inhomogeneous acoustic velocity in human tissue introduces phase aberration and degrades image quality in ultrasonic imaging systems. There have been some reports proposing correction methods for its removal. ${ }^{1-6)}$ A time-delay focusing technique was proposed by S. Flax and M. O'Donnell. ${ }^{1,2)}$ Cross-correlation of radio-frequency signals is used to estimate the time-delay between elements of a transducer array. The time-delay profile determines the optimal timedelay characteristic required to focus. Recently, M. Fink introduced a time-reversal approach. ${ }^{4-6}$ ) The pressure field detected with a set of transducer elements is digitized and stored during a time interval $T$. The pressure field is then retransmitted by the same transducers in time reversed order (last in, first out). However, there is still a need for further investigation for ultrasonic field propagating through inhomogeneous velocity layers. We obtained some analytical results for the ultrasonic field, and they show that proposed approaches as described above retain some problems to be solved.

A two-dimensional model is illustrated in Fig. 1. A transducer array is located at $z=0$ parallel to $x$-axis. A plane wave of angular frequency $\omega$

$$
e^{j(\omega t+k z)},
$$

is incident on an inhomogeneous acoustic velocity layer at $z=d$ which produces a phase variation of cosine shape in $x$ direction,

$$
e^{j(\omega t+k d)+j a \cos k_{d} x}=e^{j(\omega t+k d)} \sum_{n=-\infty}^{\infty} j^{n} J_{n}(a) e^{-j n k} \cdot x
$$

where $k=2 \pi / \lambda, \lambda$ being wave length of the incident wave and $k_{d}=2 \pi / \lambda_{d}, \lambda_{d}$ being spatial period of the phase variation. $a$ is amplitude of the phase variation.

According to Huygens's principle that the wave field in the left half space of $z<d$ is generated by the source distribution, expressed by Eq. (2), then, the pressure in a point in the left half space of $z<d$ is given by an integration over the source distribution area. At the position of the transducer array, it is,

$$
\begin{aligned}
p(x)= & \int_{-\infty}^{\infty} e^{j(\omega t+k d)+j a \cos k_{d} x} k H_{0}^{(2)}\left(k \sqrt{d^{2}+\left(x_{d}-x\right)^{2}}\right) d x_{d} \\
= & e^{j(\omega t+k d)} \sum_{n=-\infty}^{\infty} j^{n} J_{n}(a) k \int_{-\infty}^{\infty} e^{-j n k} d^{x} \\
& \cdot H_{0}^{(2)}\left(k \sqrt{d^{2}+\left(x_{d}-x\right)^{2}}\right) d x_{i l}
\end{aligned}
$$

where $\left(x_{d}, z=d\right)$ is the coordinate of the source distribution, $(x, z=0)$ is the coordinate of the array position, and

$$
k H_{0}{ }^{(2)}\left(k \sqrt{d^{2}+\left(x_{d}-x\right)^{2}}\right)
$$

represents wave propagation from a source to an element of the array. The integration of Eq. (4) gives

$$
\begin{aligned}
& p(x)=e^{j(\omega t+k d)} \sum_{n=-\infty}^{\infty} j^{n} J_{n}(a) e^{-j n k d x} k \\
& \cdot \int_{-\infty}^{+\infty} H_{0}^{(2)}\left(k \sqrt{d^{2}+\eta^{2}}\right) e^{-j n k^{n}{ }^{\eta}} d \eta
\end{aligned}
$$

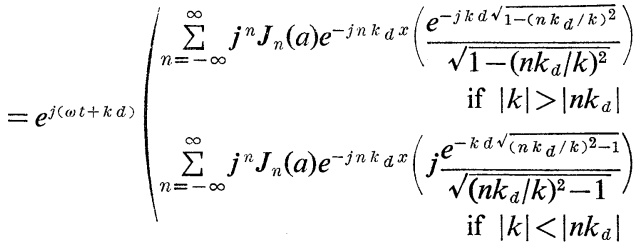

Compared with the original phase variation expressed by Eq. (2), the bracketed terms in Eq. (5) represent a new distortion of amplitude and phase due to wave propagation.

Expression of Eq. (5) is an infinite series. Only the lower order terms of the series, however, are significant and other terms can be ignored. This is because the Bessel function is very small for a sufficiently large order (this can be described with its asymptotic behavior $J_{n}(a) \approx(a / 2)^{n} / n$ ! for $\left.|n| \geq 1\right)$. Two simple cases can be discussed as follows:

Case 1: Suppose an inhomogeneous velocity layer produces a phase variation with a period smaller than wave length of ultrasonic wave, $\lambda_{d}<\lambda$ i.e., $\left|k_{d}\right|>|k|$. The second expression of Eq. (5) is used for terms of $|n| \geq 1$ and these terms approaches zero monotonically when $d$ increases. Only the term of $n=0$ remains to give a plane wavefront expression,

$$
p(x)=e^{j \omega t} J_{0}(a)
$$

This means the array at $z=0$ can not get any information about the phase variation arising at $z=d$.

Case 2: Consider $\lambda_{d}>\lambda$ and $a$ being sufficiently small so that the terms of $|n| \geq 2$ can be neglected $(a<1$ is suitable for this case). Expression (5) reduces to 


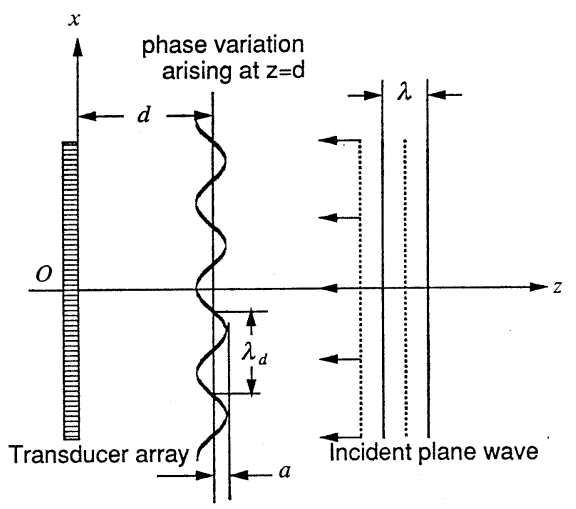

Fig. 1 A two-dimensional model. A plane wave is incident on an inhomogeneous layer at $z=d$ which produces a phase variation of cosine shape in $x$ direction, and then the wave propagates to an array at $z=0$.

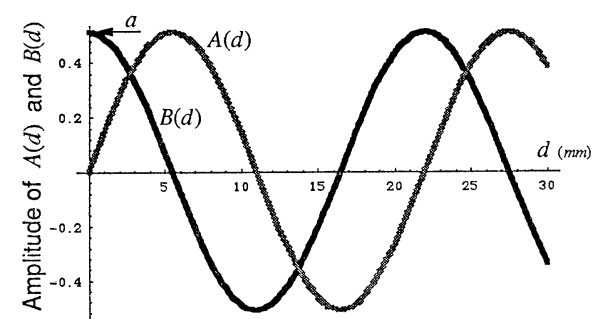

Fig. 2 Example of the amplitude variation factor $A(d)$ and the phase variation factor $B(d)$ as a function of $d$ with $a=0.5, k=14.3 \mathrm{~mm}^{-1}$, and $k_{d}=k / 5$.

$$
\begin{aligned}
p(x) \approx & e^{j(\omega t+k d)} \sum_{n=-1}^{1} j^{n} J_{n}(a) e^{-j n k_{d} x}\left(\frac{e^{j k d \sqrt{1-\left(n k_{d} / k\right)^{2}}}}{\sqrt{1-\left(n k_{d} / k\right)^{2}}}\right) \\
\approx & e^{j \omega t} J_{0}(a) \exp \left[-\frac{2 J_{1}(a)}{J_{0}(a)}\right. \\
& \left.\cdot \frac{\sin \left\{k d\left[\sqrt{1-\left(k_{d} / k\right)^{2}}-1\right]\right\}}{\sqrt{1-\left(k_{d} / k\right)^{2}}} \cos \left(k_{d} x\right)\right] \\
& \cdot \exp \left[j \frac{2 J_{1}(a)}{J_{0}(a)}\right. \\
& \left.\cdot \frac{\cos \left\{k d\left[\sqrt{1-\left(k_{d} / k\right)^{2}}-1\right]\right\}}{\sqrt{1-\left(k_{d} / k\right)^{2}}} \cos \left(k_{d} x\right)\right] \\
= & e^{j \omega t} J_{0}(a) e^{-A(d) \cos k_{d} x} e^{j B(d) \cos k_{d} x}
\end{aligned}
$$

where

$$
\begin{aligned}
A(d) & =\frac{2 J_{1}(a)}{J_{0}(a)} \frac{\sin \left\{k d\left[\sqrt{1-\left(k_{d} / k\right)^{2}}-1\right]\right\}}{\sqrt{1-\left(k_{d} / k\right)^{2}}} \\
& \approx a \frac{\sin \left\{\frac{1}{2}\left(\frac{k_{d}}{k}\right)^{2} k d\right\}}{\sqrt{1-\left(k_{d} / k\right)^{2}}}
\end{aligned}
$$

and

$$
\begin{aligned}
B(d) & =\frac{2 J_{1}(a)}{J_{0}(a)} \frac{\cos \left\{k d\left[\sqrt{1-\left(k_{d} / k\right)^{2}}-1\right]\right\}}{\sqrt{1-\left(k_{d} / k\right)^{2}}} \\
& \approx a \frac{\cos \left\{\frac{1}{2}\left(\frac{k_{d}}{k}\right)^{2} k d\right\}}{\sqrt{1-\left(k_{d} / k\right)^{2}}}
\end{aligned}
$$

are the factors involving amplitude variation and phase variation of wavefront at $z=0$ respectively. In the derivation of Eq. (7) we used a power series of exponential function for approximation in some places. Figure 2 shows examples of $A(d)$ and $B(d)$ as a function of $d$ with $a=0.5, k=14.3 \mathrm{~mm}^{-1}$, and $k_{d}=k / 5$. The disorder of the amplitude in the $x$ direction and the disorder of the phase in the $x$ direction appear reciprocally in the wave propagating path.

Our purpose is to correct the phase aberration arising at $z=d$ in an array focusing system. It is important to note the significance of this analytical results given above. As seen from the case 2, if the distance between the array and the inhomogeneous layer arising phase aberration, $d$, is not sufficiently small so that we have $A(d) \approx 0$ and $B(d) \approx a$, the wavefronts reaching the aperture of the array undergo a change of the amplitude and the phase as a function of the distance. The same statement is true for the wave moving in opposite direction transmitted from the array. An algorithm to estimate the change and then to correct the information obtained by the array are necessary for both the time-delay focusing technique and the timereversal focusing technique.

\section{References}

1) S. W. Flax and M. O'Donnell, "Phase aberration correction using signals from point reflectors and diffuse scatterers: Basic principles," IEEE Trans. Ultrason. Ferroelectr. Freq. Control 35, 758-767 (1988).

2) M. O'Donnell and S. W. Flax, "Phase aberration correction using signals from point reflectors and diffuse scatterers: Measurements," IEEE Trans. Ultrason. Ferroelectr. Freq. Control 35, 768-774 (1988).

3) G. E. Trahey, D. Zhao, J. A. Miglin, and S. W. Smith, "Experimental results with a real-time adaptive ultrasonic imaging system for viewing through distorting media," IEEE Trans. Ultrason. Ferroelectr. Freq. Control 37, 418-429 (1990).

4) M. Fink, "Time reversal of ultrasonic fieldsPart I: Basic principles;" IEEE Trans. Ultrason. Ferroelectr. Freq. Control 39, 555-566 (1992).

5) F. Wu, J. Thomas, and M. Fink, "Time reversal of ultrasonic fields-Part II: Experimental results," IEEE Trans. Ultrason. Ferroelectr. Freq. Control 39, 567-578 (1992).

6) D. Cassereau and M. Fink, "Time reversal of ultrasonic fields-Part III: Theory of the closed time-reversal cavity," IEEE Trans. Ultrason. Ferroelectr. Freq. Control 39, 579-592 (1992). 